Ignazio Cassis, Nationalrat und Vizepräsident der FMH, war von 1996 bis 2008 Tessiner Kantonsarzt. Gemeinsam mit dem Publizisten Ivo Giulietti legte er $\mathbf{2 0 0 9}$ das in italienischer Sprache verfasste Buch «ll Medico Cantonale. Dalla sua istituzione ai giorni nostri 1924-2008» vor.

Die ersten drei Kapitel des Buches schildern die Entwicklung des Gesundheitswesens im Tessin vom 1803 bis 1924 sowie die Geschichte der fünf Kantonsärzte, die dieses Amt von 1924 bis in die Gegenwart ausgeübt haben. Diese kantonsspezifischen Kapitel sind eine Fundgrube spannender historischer Dokumente, dürften aber neben Fachleuten vor allem ein regionales Publikum ansprechen.

Demgegenüber sind die «überkantonalen» Kapitel 4 und 5 sowie das Vorwort von Prof. Thomas
Zeltner, Direktor des Bundesamts für Gesundheit 1991-2009, für ein breites Publikum von Interesse. In der vorliegenden sowie der nächsten Ausgabe der SÄZ präsentieren wir unseren Leserinnen und Lesern diese Kapitel in einer deutschen Übersetzung*.

Der nachfolgende erste Teil umfasst das Vorwort sowie Kapitel 4. Im Zentrum der Ausführungen stehen die Aufgaben und die tägliche Arbeit des Kantonsarztes, unabhängig vom einzelnen Kanton: Was ist und was macht ein Kantonsarzt im Berufsalltag, mit welchen Herausforderungen ist er konfrontiert, wie sieht die Zukunft dieser Tätigkeit aus?

\section{Die Redaktion}

Die Übersetzung wurde unterstützt durch Interpharma, den Verband der forschenden pharmazeutischen Industrie in der Schweiz.

\section{Das Buch «Der Kantonsarzt» von Ivo Giulietti und Ignazio Cassis}

\section{Vorwort von Thomas Zeltner}

Das Spektrum menschlicher Krankheiten und damit verbunden das Verständnis von Gesundheit hat sich in den letzten 120 Jahren stark verändert. Vor 120 Jahren betrug die durchschnittliche Lebenserwartung in der Schweiz 41 Jahre, als Krankheits- und Todesursachen standen Infektionskrankheiten im Vordergrund.

Die Welt wurde damals, zur Zeit der Gründung der modernen Schweiz 1848, von grossen Epidemien heimgesucht. Sechs grosse Cholera-Epidemien erreichten im 19. Jahrhundert Europa. So forderte diese Krankheit ab 1884 innerhalb von drei Jahren in Frankreich, Italien und Spanien mehr als 200000 Todesopfer. Im Jahre 1886 starben in der Schweiz 182 Personen an Pocken, und 9 Jahre zuvor hatte der Flecktyphus auf dem Balkan gewütet. Doch der Schutz der Gesundheit der Bevölkerung stand in den Gründerjahren der Eidgenossenschaft nicht im Vordergrund. Die schweizerische Bundesverfassung gestand dem Bundesstaat im Bereich der Gesundheit nur beschränkte Kompetenzen zu: Das schweizerische Gesundheitssystem war und ist bis heute in einem hohen Mass von kantonaler Autonomie geprägt.

An den Cholera- und Pockenepidemien litt auch das Tessin. Die Epidemien wurden in der Regel aus der nahen Lombardei oder Norditalien eingeschleppt. Als sich 1867 die Cholera zum letzten Mal im Tessin ausbreitete, erkrankten 221 Menschen und 122 starben. Daraus resultierte 1870 ein Gesetz, das die Gründung obligatorischer Kreisphysikate vorschrieb. Diese sollten eine kostenlose Behandlung gewährleisten, mit Ausnahme grösserer chirurgischer Eingriffe. Man wollte eine Behandlung garantieren, die sowohl im entscheidenden Augenblick als auch generell und zu beschei- denen Preisen greifen konnte und auch denen zugute kam, die an entlegenen Orten wohnten. Dies entsprach im Grunde dem, was rund 100 Jahre später in der Form eines gesetzlich vorgeschriebenen, gleichen Zugangs für alle zur Gesundheitsversorgung mit umfassendem Leistungskatalog entstand.

Obwohl also das schweizerische Gesundheitssystem stark kantonal geprägt war, zeigte sich in bestimmten Bereichen früh der Bedarf nach Regelung und Koordination auf Bundesebene. Nämlich dort, wo der zunehmende interkantonale und internationale Verkehr sowie der technische und wissenschaftliche Fortschritt wirkten. So zeigte im Jahr 1877 die Einführung der Freizügigkeit der Medizinalpersonen in der Schweiz - mit der Einführung eines eidgenössischen Diploms für Ärzte, Tierärzte und Apotheker - zwar klar, dass der kurative medizinische Teil als erstes geregelt werden würde. Doch die Schaffung eines Gesundheitsamtes drängte sich bald darauf auf, als nach mehreren internationalen Konferenzen die Bekämpfung der Pockenepidemien unumgänglich wurde und ein erstes Epidemiengesetz 1882 in der Volksabstimmung scheiterte. Die Einführung des Epidemiengesetzes 1886 und die weiterhin stark grassierenden Pockenepidemien bewegten den Bundesrat schliesslich dazu, mit Beschluss vom 28. Juni 1893 das Schweizerische Gesundheitsamt zu gründen. Dieses bestand damals aus einem Direktor, einem Adjunkt und einem Kanzlisten.

Aus der Gründungszeit des BAG stammen also die wichtigen Aufgaben der Epidemienbekämpfung und mit der Einführung des Lebensmittelgesetzes 1905 die Lebensmittelkontrolle. Das Bundesgesetz vom 18. Dezember 1970 über die Bekämpfung übertragbarer Krankheiten des Menschen (Epidemiengesetz, EpG) ist im Nachgang der schweren Typhusepidemie in Zermatt im Jahre 1963 mit mehr als 400 Erkrankungen und mehreren Todesfällen, darunter auch vielen ausländischen Touristen, entstanden. Dieses Gesetz hat 


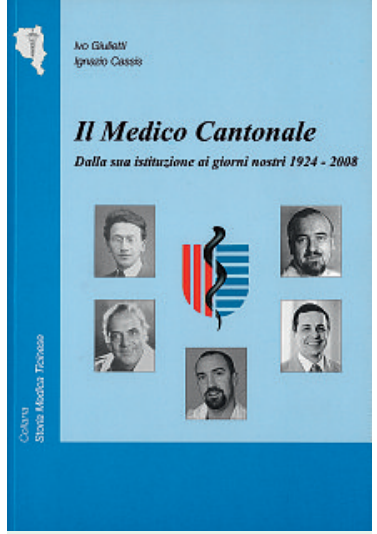

Ivo Giulietti, Ignazio Cassis II Medico Cantonale Dalla sua istituzione ai giorni nostri 1924-2008.

Taverne: Storia Medica Ticinese; 2009.

240 Seiten. Fr. 48.-. ISBN 978-88-902131-5-9 der Bekämpfung übertragbarer Krankheiten einen aktuellen gesetzlichen Rahmen gegeben und mit dem Amt der Kantonsärztin/des Kantonsarztes ein wichtiges Bindeglied zwischen Bund und Kantonen sowie zwischen der öffentlichen und individuellen Gesundheit geschaffen.

Heute steht die epidemiologische Beobachtung übertragbarer Krankheiten immer mehr im Zentrum der Aktivitäten. Denn seit der Mitte des 20. Jahrhunderts ist mit der zunehmenden Mobilität der Bevölkerung (mehr Reisende und höhere Reisegeschwindigkeiten) auch die Gefahr der Verbreitung von Infektionskrankheiten weiter gestiegen. So prägt die globale Vernetzung und Verdichtung unserer Welt in wachsendem Masse auch die schweizerische Gesundheitspolitik. Der intensive Reise- und Handelsverkehr mit dem Ausland zwingt zur ständigen Abstimmung unserer Gesetzgebung mit jener unserer Nachbarländer, insbesondere mit jener der Europäischen Gemeinschaft. Was sich im 19. und 20. Jahrhundert kantonal und dann national entwickelt hat, nämlich die Übernahme von Verantwortung durch den Staat und das Einrichten von staatlichen Institutionen, wiederholte sich in der Europäischen Union 2005 mit der Gründung des Europäischen Zentrums für die Prävention und Kontrolle von Krankheiten (ECDC) als direkte Folge der
SARS-Krise. Die geographische und wirtschaftliche Lage der Schweiz macht eine zunehmend enge und effiziente Zusammenarbeit mit den EU-Institutionen auch im Gesundheitsbereich unabkömmlich. Der freie Personenverkehr im europäischen Raum, das Wegfallen der innereuropäischen Grenzen sowie der intensive Austausch von Produkten und Lebensmitteln bergen immer neue und steigende Gesundheitsgefahren.

Gleichzeitig steigt aber berechtigterweise der Anspruch der Bevölkerung nach immer mehr Sicherheit und Verminderung von Gesundheitsrisiken. Neben den enorm steigenden Kosten in der kurativen Gesundheitsversorgung sind dies die grossen Herausforderungen im Bereich der öffentlichen Gesundheit, welche nur im internationalen Verbund angegangen werden können. Nichtsdestotrotz müssen sich aber in der Schweiz auch die Kantone und damit die Kantonsärzte als die peripheren Hüter der öffentlichen Gesundheit diesen Herausforderungen stellen und sie als Chance nutzen. Ein Rückblick in die Vergangenheit, wie ihn das vorliegende Buch bietet, ist dabei immer eine hervorragende Gelegenheit, Verständnis zu wecken, Reflexionen anzustellen und Lehren aus der Geschichte zu ziehen, um offen zu sein für alte und neue Herausforderungen im Gesundheitsbereich.

\section{Die Tätigkeit des Kantonsarztes*}

\section{Ignazio Cassis}

Dr. med., Nationalrat und Vizepräsident der FMH

* Übersetzung des Kapitels 4 aus dem Buch von Ivo Giulietti und Ignazio Cassis «Il Medico Cantonale».

Korrespondenz:

Dr. med. Ignazio Cassis

FMH

Elfenstrasse 18

CH-3000 Bern 15

ignazio.cassis@parl.ch

\section{Einleitung}

Über die Einrichtung des Kantonarztes ist wenig bekannt. Seitdem ich Ende 1996 meine Tätigkeit aufgenommen hatte, hörte ich ständig die gleiche Frage: «Was macht eigentlich ein Kantonsarzt?» Ich gebe zu, dass mich die Beantwortung dieser Frage in eine gewisse Verlegenheit brachte, weil das Aufgabenspektrum des Kantonsarztes so breit gefächert und in ständiger Entwicklung begriffen ist, dass ich Schwierigkeiten hatte, darauf eine einfache und treffende Antwort zu finden. Ich zitierte dann die entsprechenden Gesetzesartikel und lieferte, weil diese doch etwas allgemein und abstrakt sind, einige Beispiele aus meinem Alltag dazu. Schwieriger ist es dagegen, die umfangreichen und oft nur vage umschreibbaren Tätigkeitsbereiche in einem einzigen Satz, einer Formel, zusammenzufassen. Ein Freund meinte einmal: «Na, ist doch ganz einfach: Der Arzt behandelt einzelne Patienten; der Kantonsarzt behandelt alle Patienten eines Kantons.» Obwohl diese Bemerkung scherzhaft gemeint war, enthält sie doch viele Elemente einer korrekten Antwort.

Die Frage hat mich während meiner ganzen elf Jahre als Kantonsarzt begleitet. Es mag wohl sein, dass ich im Laufe der Zeit die Tätigkeitsbereiche des Amtes, das ich führte, definiert, geordnet und quantifiziert habe; das Fehlen eines klaren Berufsprofils und einer historischen Identität dieser staatlichen Institution hat mir dabei jedoch stets zu schaffen gemacht. Der Gedanke, dieser Frage ein Buch zu widmen, das die Entstehung und Entwicklung dieser Einrichtung zum Gegenstand haben und einige Denkanstösse zur praktischen Amtsausübung liefern sollte, ist mir bereits früh gekommen, nämlich schon vor der Jahrtausendwende. Meinem Kollegen Mario Lazzaro damals stellvertretender Kantonsarzt - und mir war nämlich klar geworden, dass das berufliche Profil dieser Funktion mit Blick auf ihre weitere Entwicklung und im Interesse künftiger Amtsinhaber schärfer umrissen werden musste. Dennoch fand ich erst unmittelbar vor meinem Ausscheiden aus dem Amt die innere Bereitschaft und die finanziellen Mittel zur Realisierung dieser Idee. Die unverhoffte Begegnung mit Ivo Giulietti, einem Autodidakten der Medizingeschichte des Kantons Tessin, hat dazu ein Übriges getan. Er zeigte sich von der Idee sofort begeistert und verbrachte ungezählte Tage mit Recherchen in diversen Archiven, um die Geschichte des Kantonsarztes vor dem Hintergrund der Entwicklung des Gesundheitswesens in den letzten zwei Jahrhunderten zu rekonstruieren. Und er wartete geduldig, bis ich zwischen meinen unterschiedlichen Verpflichtungen die erforderliche Zeit fand, meinen Teil an der schriftlichen Ausarbeitung beizusteuern. Ohne Ivo Giulietti wäre 
das vorliegende Buch nie entstanden; ihm gebührt daher mein herzlicher Dank!

\begin{abstract}
Identität
Vorbehaltlos stimme ich dem ehemaligen Waadtländer Kantonsarzt Jean Martin zu [1], der meinte, der Kantonsarzt sei das, was im angelsächsischen Sprachraum als «Jack of all trades», «Mädchen für alles» bezeichnet werde, also einer, der in allen Bereichen massgeblichen Einfluss hat. Oder, einfacher ausgedrückt, gemäss der Definition von Jean-Luc Baierlé, Kantonsarzt im Kanton Jura, ein «Generalist der Volksgesundheit» [2]. Er muss in erster Linie die grösseren Zusammenhänge im Blick behalten und darf sein Augenmerk nicht auf einen bestimmten Fachbereich beschränken. Er muss in der Lage sein, komplexe Probleme aufzuschlüsseln und aus unterschiedlichen Perspektiven zu analysieren, um sie anschliessend in geordneter und verständlicher Form wieder zusammenzusetzen, damit die angemessenen Massnahmen zu ihrer Lösung eingeleitet werden können. Was ihn also auszeichnet, ist seine Analyse- und Synthesefähigkeit.
\end{abstract}

\section{Ständig hörte ich die gleiche Frage: «Was macht eigentlich ein Kantonsarzt?»}

Bereits in den Sechzigerjahren des vergangenen Jahrhunderts hat Marcel Cevey, damals Kantonsarzt im Kanton Waadt, zusammengefasst, welche Fähigkeiten ein Kantonsarzt mitbringen muss: «... le médecin cantonal doit être à la fois un scientifique capable de comprendre les besoins d'une faculté, un praticien apte à saisir les problèmes concrets de la médecine extra-hospitalière, un épidémiologue averti, un routinier des problèmes d'assurances, un pédagogue émérite, un juge impartial sachant faire respecter le code de déontologie, un orateur à ses heures, un diplomate avisé, un politicien accompli...» [3]. Von allem ein bisschen also, mit dem notwendigen Blick auf das Ganze.

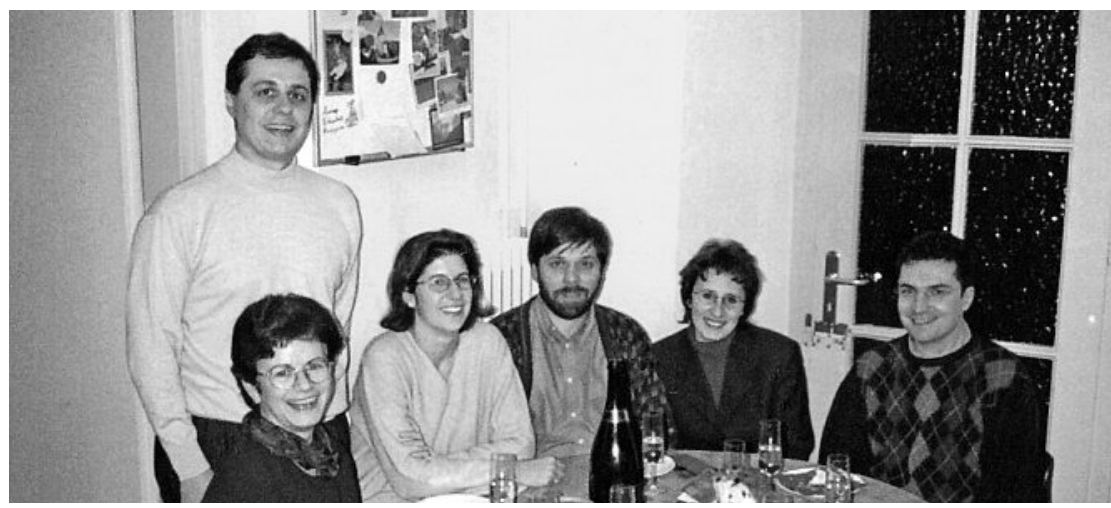

Ignazio Cassis (stehend) im Jahr 1999, drei Jahre nach Beginn seiner Tätigkeit als Kantonsarzt mit Kollegen.

\section{Zwischen Medizin und Politik}

Durch seine Rolle als verbindendes Glied zwischen Medizin und Politik kommt dem Kantonsarzt eine wesentliche vermittelnde Funktion für deren gegenseitige Verständigung zu. Viele seiner Tätigkeiten haben daher etwas mit dem Begriff «Übersetzen» zu tun. Der Kantonsarzt fungiert als Übersetzer bzw. Dolmetscher zwischen zwei Welten - Politik und Medizin -, die nur schwer miteinander in Dialog treten oder einander gar ignorieren, aber stets gefordert sind, miteinander $\mathrm{zu}$ kommunizieren, $\mathrm{zu}$ interagieren und auf diese Weise zu Lösungen im Interesse des «Gemeinwohls» zu gelangen. Die Berufslogik des Arztes basiert einerseits auf der jahrtausendealten Tradition der abendländischen Medizin mit ihren wissenschaftlichen Grundlagen und spezifischen Regeln (deontologischer Kodex), andererseits auf der langen Tradition der autonomen Berufsausübung. Die politische Logik setzt das öffentliche bzw. das Allgemeininteresse ins Zentrum ihres Handelns; sie betrachtet den Gesundheitssektor als einen gesellschaftlich wichtigen Sektor, jedoch nicht als den einzigen. Ihr Ziel ist es, jedem Bürger den Zugang zu einer qualitativ hochwertigen und finanziell tragbaren medizinischen Versorgung zu garantieren. Sie ist ständig bemüht, ein ausgeglichenes Verhältnis zu finden zwischen den in diesen und anderen sozialen Sektoren (Bildung, Transport, Sicherheit, Wohnraum, Soziales usw.) investierten Ressourcen.

So wirtschaftlich stark die Schweiz auch sein mag, geht der Politiker doch stets von der Vorstellung aus, dass Ressourcen zwangsläufig begrenzt sind und das Interesse des einzelnen Patienten daher nicht immer Vorrang vor dem Gemeininteresse haben kann. Der einzelne Patient bringt hierfür jedoch nicht immer Verständnis auf und verlangt vom Arzt, sein «Möglichstes» zu tun, um seinen Bedürfnissen (oder Wünschen) entgegenzukommen. Für den modernen Patienten der letzten Jahrzehnte hat Gesundheit keinen Preis, auch wenn sie unleugbar mit Kosten verbunden ist. Wir Ärzte haben in der Ausbildung gelernt, nach der Maxime «Salus aegroti suprema lex» zu handeln: das Wohl des Kranken ist oberstes Gesetz. Von juristischer Seite wird uns sogar nahegelegt, diese umzuformulieren in «Voluntas aegroti suprema lex», der Wille des Kranken ist oberstes Gesetz - mit der Begründung, dass das ethische Prinzip der Selbstbestimmung über allem stehe. Dadurch entsteht zwangsläufig eine Spannung zwischen Individuum und Kollektiv, zwischen Medizin und Politik. Ciceros Maxime «Salus populi suprema lex esto», das Heil des Volkes sollte oberstes Gesetz sein, war konditional formuliert. Das ist insofern von grosser Bedeutung, als das Prinzip auf diese Weise in Bezug gesetzt wird zu den realen Möglichkeiten der Gesellschaft. Der Konditional ging mit der Umwandlung des Satzes irgendwann verloren.

Zwei Logiken also - die ärztliche und die politische-, deren Verständigung untereinander nicht ganz problemlos ist. Als Kantonsarzt habe ich viel Energie darin investiert, der einen Seite die Perspektive der 
anderen zu vermitteln und umgekehrt. Daraus wird ersichtlich, wie dringend notwendig es ist, an den eigenen pädagogischen Fähigkeiten zu feilen, wie dies Marcel Cevey bereits vor über vierzig Jahren gut beschrieben hat. dass er es nur selten schafft, sein ehrgeiziges Tagesprogramm einzuhalten; der Einfluss der Politik zwingt ihn, auf nicht immer feine Art, in Bezug auf die Führung der eigenen Mitarbeiter zu ständigen inhaltlichen und terminlichen Anpassungen.

\section{Der Kantonsarzt fungiert als Dolmetscher zwischen zwei Welten - Politik und Medizin -, die nur schwer miteinander in Dialog treten}

\section{Ein Staatsbediensteter}

Trotz der weitreichenden Autonomie, über die er verfügt, ist der Kantonsarzt nicht per definitionem unabhängig. Er steht im Dienst der Kantonsverwaltung. Die Ärzteschaft - deren Handlungsautonomie in der jeweiligen Kultur verankert ist - findet sich mit dieser Tatsache bisweilen nur schwer ab. Und auch für den jungen Kantonsarzt ist es nicht immer einfach, sich an diesen Gedanken zu gewöhnen. Als leitender Angestellter der Gesundheitsbehörde muss er eng mit anderen Mitarbeitern in ebenfalls leitenden Positionen zusammenarbeiten, z. B. Juristen, Ökonomen, Soziologen, Verwaltungsfunktionären und, nicht zuletzt, auch mit Politikern. Seine berufliche Erfüllung hängt also von seiner Fähigkeit ab, konstruktiv mit anderen «Fachkulturen» zu interagieren oder, besser noch, im Interesse seiner persönlichen Weiterentwicklung und der Erweiterung seines kulturellen Horizonts davon zu profitieren. Eine wesentliche Voraussetzung für die erfolgreiche Amtsausübung ist, dass der Kantonsarzt es versteht, unter Berücksichtigung der entscheidenden Rolle der Gesundheitsbehörde diesen interdisziplinären Rahmen voll auszuschöpfen.

\section{Diplomatisches Geschick und Frustrationstoleranz}

Häufig übernimmt der Kantonsarzt den Vorsitz in Arbeitsgruppen und Ausschüssen, die mit der Untersuchung und Beurteilung von Themen beauftragt werden, bei denen einander entgegengesetzte Interessen im Spiel sind. Hier für einen produktiven, geordneten Ablauf zu sorgen, erfordert viel diplomatisches Geschick. Wichtig ist auch die Fähigkeit, seine eigenen Vorstellungen vertreten zu können, ohne sich dabei auf seine Meinung zu versteifen. Ferner ist die Fähigkeit zur Analyse und Synthese unerlässlich, um sich nicht im Nebensächlichen zu verlieren und dabei das eigentliche Ziel zu verfehlen. Auch die enge Zusammenarbeit mit dem Departementsvorsteher [4], der vom Volk gewählt und bisweilen durch politische Entwicklungen motiviert ist, ist keine reine Selbstverständlichkeit: Orientierungswechsel, plötzliches Drängen oder Bremsen, Auf- oder Abstieg hoher Amtsträger sind allesamt Ereignisse, die starke Nerven und eine hohe Frustrationstoleranz erfordern. Kennzeichnend für die Arbeit des Kantonsarztes ist auch die Tatsache,

\section{Beratung}

Die Aufgaben des Kantonsarztes erschöpfen sich jedoch nicht in der praktischen Umsetzung von Gesetzen des Bundes (Epidemien, Betäubungsmittel, Krankenversicherung, Reproduktionsmedizin, Schwangerschaftsabbruch) oder der Kantone (hauptsächlich Gesundheitsgesetz). Ein arbeitsintensiver, aber gleichzeitig angenehmer Aspekt ist der tägliche Kontakt mit den im Kanton praktizierenden Ärzten, den Mitarbeitern aller Dienste und Einrichtungen des Gesundheitswesens, den Schulärzten (über den schulmedizinischen Dienst) sowie, im Rahmen des Möglichen, den Bürgern selbst. Dabei geht es um Beratungsgesuche zu juristischen Aspekten, ethischen Fragen und komplexen sozialen Problemen, die einer Gesamtbetrachtung bedürfen und eine gute Kenntnis der Dienste und Einrichtungen des Kantons voraussetzen. Häufig hilft der Kantonsarzt seinem Gegenüber dabei, Klarheit zu schaffen, Prioritäten zu setzen und schliesslich zu einer tauglichen Lösung zu gelangen. Bisweilen kommt es auch vor, dass ein Gesprächspartner an eine andere, für das jeweilige Problem zuständige «Stelle» weitergeleitet werden muss. Hier sind viel gesunder Menschenverstand und natürlich auch Erfahrung gefragt. Um mit Oscar Wilde zu sprechen: «Erfahrung ist der Name, den die Menschen ihren Irrtümern geben.» Auch der Kantonsarzt ist in seinem Urteil nicht unfehlbar. Wichtig ist aber, dass er Fehler ohne Angst vor Verurteilung zugeben kann, sowohl sich selbst gegenüber als auch gegenüber den eigenen Mitarbeitern und dem jeweiligen Betroffenen. Dem, der ein verantwortungsvolles Amt bekleidet, ist Bescheidenheit eine Zier.

\section{Kompetenzen nicht überschreiten}

Eine weitere Voraussetzung, deren Bedeutung mir im Laufe der Zeit bewusst geworden ist, hängt mit der Frage zusammen: «Wer ist in dieser Sache der richtige Ansprechpartner?» Bevor man eifrig und mit gezücktem Schwert dem Betroffenen zu Hilfe eilt, muss man sich stets die Frage stellen: «Wer ist hier zuständig, in wessen Verantwortlichkeitsbereich fällt dieses Problem?» Dadurch vermeidet man nicht nur, mit seinen Hilfsbemühungen zu scheitern, sondern spart auch Zeit - die des Hilfesuchenden und die eigene. Eine von diesem Problem besonders betroffene Kategorie sind die Hausärzte. Sie sind bei Problemen häufig die erste 
Anlaufstelle ihrer Patienten und neigen in guter Absicht dazu, sich zu deren Anwälten zu machen und sich an der Klärung komplizierter verwaltungstechnischer und juristischer Fragen zu versuchen. Nur sind sie eben keine Anwälte, und nur wenige verfügen überhaupt über gute Rechtskenntnisse. Angesichts der steigenden Zahl von Konflikten in den Bereichen Arbeit, Versicherung, Ehe und Familie, Sorgerecht, Verkehr usw. können einige der Versuchung, «Recht zu sprechen», nicht widerstehen. Mitunter habe ich dabei auch grobe Fehler erlebt; den Schaden hatten am Ende die betroffenen Patienten. Ich habe mich stets bemüht, in solchen Fällen die Ärzte davon zu überzeugen, sich nicht auf fremdes Territorium zu begeben, sondern, wie der Schuster, «bei ihren Leisten» zu bleiben, ihre Patienten selbstverständlich in psychischer Hinsicht zu unterstützen, ohne jedoch die Rolle des Anwalts, Richters, Sozialarbeiters oder was auch immer zu übernehmen. Viele waren mir im Nachhinein dafür dankbar.

\section{Richter}

Die Rolle des Richters stellt für jeden Kantonsarzt eine bedeutende Herausforderung dar. Wie kann man einerseits ein guter Ansprechpartner, ein verständnisvoller, diskreter, vertrauenswürdiger Berater sein und andererseits bei Problemen als Autorität auftreten, beispielsweise, indem man einen Arzt dazu zwingt, Bereitschaftsdienst zu leisten, oder ihm sogar nahelegen muss, einem Arzt seine Zulassung zur Berufsausübung zu entziehen [5]? Dabei können peinliche Rollenkonflikte entstehen, die eine sorgfältige Abwägung erfordern. Andererseits ist es in einem relativ kleinen Wirkungskreis wie dem unseren nicht vorstellbar, beide Rollen voneinander zu trennen. Dem stehen Zwänge finanzieller Art entgegen, aber auch die Frage nach der kritischen Masse. Jeder Kanton hat seine eigene, mehr oder weniger gelungene Lösung, die mehr oder weniger im Einklang mit den lokalen Traditionen steht. Im

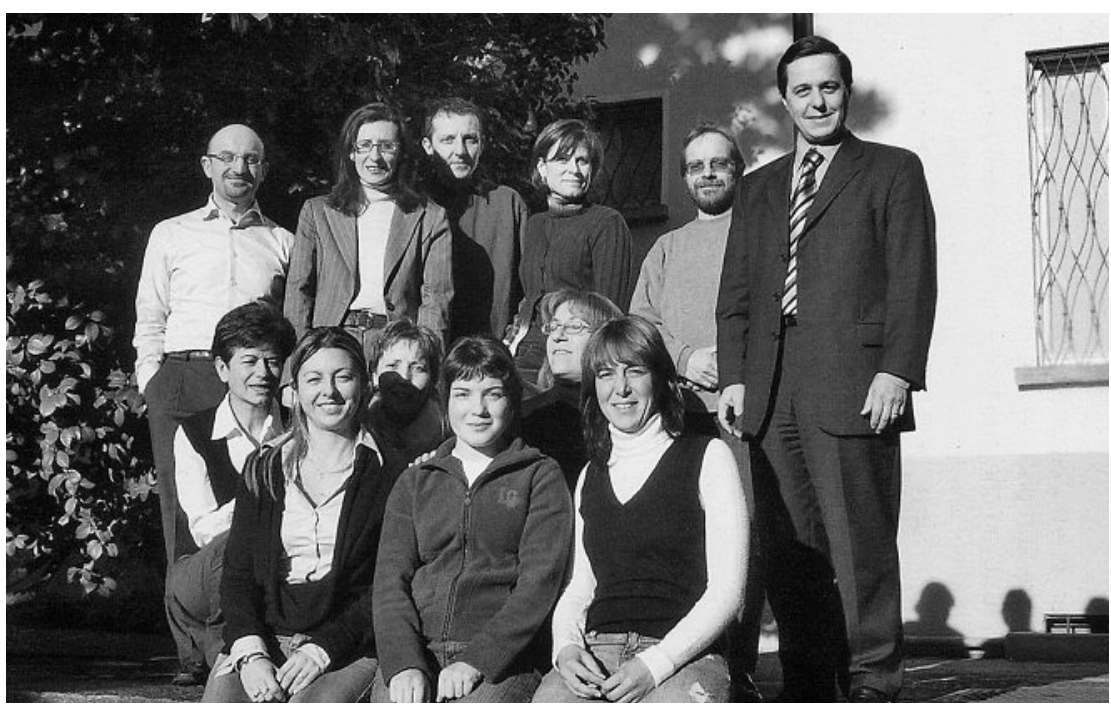

Aufnahme aus dem Jahr 2006. Zwei Jahre später beendete Ignazio Cassis seine Tätigkeit als Kantonsarzt.
Tessin wird nach alter Gewohnheit die formale Aufgabe, einen Fall einzuleiten und einen offiziellen Entscheid zu fällen, nicht dem Kantonsarzt, sondern einer anderen Stelle übertragen [6]. Der Kantonsarzt tut sicher gut daran, rasch zu begreifen, dass Gesetz, Recht und Gerechtigkeit nicht dasselbe bedeuten eine überraschende Erkenntnis für einen Idealisten. Das Recht soll die Stimme des Staates ausdrücken, der das unumschränkte Gewaltmonopol innehat und für die Rechtsprechung Sorge trägt. Das Recht selbst basiert jedoch auf Gesetzen, die Unvollkommenheiten aufweisen, und auf Richtern, die wie alle Menschen fehlbar sind.

\section{Audiatur et altera pars}

Abwägung, Verhältnismässigkeit und Ausgewogenheit sind wesentliche Voraussetzungen für die Tätigkeit des Kantonsarztes; das gilt sowohl für Entscheidungen politischer Natur als auch dort, wo es darum geht, bei konkreten Problemen Lösungen anzubieten. Nicht selten überwältigt ein Hilfesuchender - Arzt, Verwalter, Dozent oder Bürger - den Kantonsarzt am Telefon mit einer emotional aufgeladenen Geschichte, gespickt mit augenscheinlichen Ungerechtigkeiten, die an den Gerechtigkeitssinn appellieren und nach Rache schreien. Die ungünstigste Reaktion wäre dann, sich von einer derartigen Aufregung anstecken zu lassen und zum Kampf zu schreiten. Sehr oft sind die Verhältnisse komplexer, als sie auf den ersten Blick scheinen mögen. Die Probleme werden vom Betroffenen in einem Kontext aus Vorurteilen oder persönlichen Konflikten erlebt, und dies wirkt sich natürlich auf seine Darstellung der Dinge aus. Um hier weiterzukommen, ist es von grundsätzlicher Bedeutung, auch die andere Seite zu Wort kommen zu lassen: «Audiatur et altera pars», lautet das berühmte Zitat von Seneca. Fast immer ergibt sich dabei ein von der ursprünglichen Schilderung erheblich abweichendes Bild der Situation, und in der Folge fallen die beschreitbaren Lösungswege nicht nur angemessener, sondern auch wirksamer aus.

Bei politischen Entscheidungen ist die Kompromissfindung Teil der eidgenössischen Tradition, die Konflikte nur schwer toleriert. Bei ausgewogenen Lösungen besteht jedoch immer die Gefahr, dass streitbare Geister sich nicht damit zufriedengeben; den einen ist die Entscheidung zu lasch, den anderen zu streng. Als Kantonsarzt - und das gilt in gleichem Masse für viele staatliche Ämter - muss man lernen, dies zu akzeptieren, und darf nicht versuchen, es allen recht machen zu wollen!

\section{Journalisten und Medien}

Wir leben in einer Informationsgesellschaft. Für den Kanton Tessin gilt dies angesichts der dortigen hohen Dichte an Kommunikationsmitteln in noch grösserem Masse. Es vergeht kein Tag, an dem der Kantonsarzt nicht von einem Journalisten in der einen oder anderen Angelegenheit kontaktiert wird, die nicht immer direkt mit dem Gesundheitswesen zu tun hat. Bedeu- 
tende Nachrichten gibt es nur selten, Nachrichten allgemeiner Art dagegen jede Menge. Entziehen kann man sich dem Spiel aber nicht: Journalisten leben auf dem Sprung und erwarten, dass die Vertreter des Staates permanent zu ihrer Verfügung stehen. Wer sich ihnen verweigert - sei es, weil das betreffende Thema gerade nicht oben auf der Tagesordnung steht, sei es aus Ungehaltenheit über die Beharrlichkeit des Journalisten bei reinen Klatschthemen - verliert damit am Ende noch mehr Zeit. Einmal beklagte sich ein Sonntagsblatt schriftlich beim Staatsrat darüber, dass ich ein Ereignis nicht hatte kommentieren wollen. Die Kantonsregierung leitete die Anfrage an mich weiter mit der Bitte um Stellungnahme und erteilte nach einer anschliessenden Besprechung dem Chefredaktor eine sehr diplomatisch formulierte Antwort. Eine äusserst frustrierende Angelegenheit, die mich insgesamt erheblich mehr Zeit kostete als die fünf Minuten, die ein Telefonat mit dem Journalisten gedauert hätte. Ausserdem war mir bewusst, dass die Regierung - die mir deswegen übrigens keine Vorwürfe machte - sich in einer peinlichen Lage befand, da sie kein gespanntes Verhältnis zur Presse haben wollte. Politik und Medien sind eng miteinander verwoben und aufeinander angewiesen. Das habe ich bei dieser Gelegenheit erst richtig begriffen.

\section{Politiker gehen davon aus, dass Ressourcen begrenzt sind und das Interesse des Patienten nicht immer Vorrang vor dem Gemeininteresse haben kann}

\section{Never complain, never explain}

Ich lernte also, für Anfragen von Journalisten stets erreichbar zu sein, auch in Fällen, in denen ich dies für Zeitverschwendung erachtete. Der Umgang mit Journalisten ist nicht immer einfach. Ihnen mit Voreingenommenheit zu begegnen, wäre ebenso verkehrt, wie Naivität an den Tag zu legen. Man muss sich ihre Logik aneignen und mitspielen, ohne sich dabei über Gebühr «ausnutzen» zu lassen. Eine solche Haltung wird in der Regel geschätzt und dankbar registriert, und daraus entwickelt sich ein Klima gegenseitigen Vertrauens. Aufpassen muss man insbesondere bei suggestiven Fragen und Fangfragen. Im Zweifelsfall bittet man bei gedruckten Beiträgen darum, das Interview vor der Veröffentlichung noch einmal durchlesen zu dürfen. Am tückischsten sind Auftritte in Fernsehen und Radio: bei «Liveübertragungen» wird in Echtzeit gespielt. Das erfordert, unabhängig von den gestellten Fragen, hohe Konzentration und absolute Klarheit bei den Botschaften, die man übermitteln will. Manchmal schafft man es, bis zum Schluss optimal die Balance zu halten. Und sobald man nicht mehr «auf Sendung» ist und in der informelleren Atmosphäre die Spannung von einem abfällt, lässt man sich eine unbedachte
Äusserung entschlüpfen - der klassische «Satz zu viel». Natürlich taucht genau dieser Satz am nächsten Tag in den Nachrichten auf. Dann Himmel und Hölle in Bewegung zu setzen und Dementis zu veröffentlichen, ist sinnlos: das Kind ist schon in den Brunnen gefallen, und jede weitere Äusserung verschlechtert nur die Situation. In solchen Momenten halte ich mich strikt an Churchills Devise «Never complain, never explain» und halte meinen Mund. Der Spruch bedeutet in etwa «Nicht jammern und keine Erklärungen abgeben» - auf gut Deutsch: Was man nicht sagt, kann nicht gegen einen verwendet werden! Einen Tag später ist die ganze Aufregung meist schon Schnee von gestern, und eine andere Nachricht beherrscht die Schlagzeilen.

Der Kantonsarzt muss sein Verhältnis zu den Medien umsichtig und korrekt, aber auch authentisch und spontan gestalten. Die Kommunikation ist für eine effektive Amtsführung von grossem Wert; die Pflege einer guten Beziehung zu den Pressevertretern gleicht daher der Instandhaltung einer guten Maschine.

\section{Das Team}

Der Kantonsarzt ist nicht allein. Ohne ein Team von zuverlässigen und motivierten Mitarbeiterinnen und Mitarbeitern wäre seine Arbeit absolut undenkbar. Das Team besteht je nach Zeitpunkt und laufenden Projekten aus 10 bis 15 Personen. Zusammen bilden diese das Kantonsarztamt, eine Verwaltungseinheit, der der Kantonsarzt vorsteht und die in das Organigramm des Gesundheits- und Sozialdepartements eingegliedert ist. Tatsächlich ist, wenn vom Kantonsarzt als Einrichtung die Rede ist, eigentlich das Kantonsarztamt gemeint - ähnlich, wie man auch vom «staatlichen Preisaufseher» spricht oder vom «Datenschutzbeauftragten». Und ein Team muss geführt, motiviert, geformt und - wenn nötig - auch unterstützt werden. Eine zu Unrecht oft als sekundär angesehene Aufgabe, der in Wirklichkeit jedoch entscheidende Bedeutung zukommt. Eine Aufgabe, die viel Zeit, Hingabe, Bereitschaft, Verständnis und Aufmerksamkeit erfordert. Mit einem lahmen Pferd gewinnt man kein Rennen. Vom stellvertretenden Amtsvorsteher, der rechten Hand des Kantonsarztes, über die wissenschaftlichen Mitarbeiter bis hin zu den Sekretariatsangestellten und Praktikanten leisten alle ihren Beitrag an der Abwicklung der täglichen Aufgaben - von den einfacheren bis hin zu den anspruchsvolleren und kritischen.

Das Führen eines «kleinen bis mittelständischen Unternehmens» im Bereich der öffentlichen Verwaltung ist eine Herausforderung, deren Behandlung ein eigenes Kapitel wert wäre. Im Gegensatz zu einem Privatunternehmen sind die Mitarbeiter hier nicht Angestellte des Chefs, sondern des Staates. Ihr Angestelltenverhältnis wird nicht durch einen flexiblen privatrechtlichen Arbeitsvertrag geregelt, sondern durch die strengen Gesetze und Verordnungen, die das Beamtenstatut definieren. Das ist kein formaler, sondern ein 
substantieller Unterschied. Ich selbst habe Jahre gebraucht, um mir der eigentlichen Bedeutung dieses Umstands bewusst zu werden. Die «Macht» des Amtsvorstehers ist demnach recht beschränkt; er kann Mitarbeiter nicht einfach einstellen und entlassen, er kann weder leistungsorientierte Gehälter festlegen noch Sonderzuwendungen oder Sanktionen beschliessen, ohne mühselige und oft unüberwindbare verwaltungstechnische und juristische Prozeduren (häufig bis vor das Bundesgericht) auf sich $\mathrm{zu}$ nehmen, deren politische Auswirkungen - Beschlussfassungen des Parlaments, Druck auf die Regierung - einen Bumerangeffekt erzeugen, der schliesslich auf ihn selbst zurückfällt. Der Amtsvorsteher hat nicht die Möglichkeit, finanzielle Anreize zur Motivierung der eigenen Mitarbeiter zu schaffen; alles, sein Einfluss in dieser Hinsicht, ist unmittelbar an seine Person, seine Einstellung, sein Motivierungsvermögen gebunden. Jemand hat einmal gesagt, dass das erfolgreiche Führen eines Amtes innerhalb der öffentlichen Verwaltung die Feuerprobe für jeden leitenden Angestellten darstellt - ich meine, dass er damit recht hatte. Leicht kann man dabei ins Schlittern geraten und auf die Nase fallen, so viele Fallstricke und Fettnäpfchen lauern in den «Gängen des Palastes» [7].

\section{Der Patient bringt für begrenzte Ressourcen nicht immer Verständnis auf und verlangt vom Arzt,} das «Möglichste» zu tun

Der Mitarbeiterstab ähnelt in vielem einer Familie. Man verbringt viele Stunden zusammen, setzt sich miteinander für etwas ein, hat gemeinsame Erfolgserlebnisse, teilt aber auch Frustration und Ärger. Hier die Rolle des Amtsvorstehers zu übernehmen, ist keine Kleinigkeit - nicht etwa, weil die Verwaltung eines Budgets oder das Erreichen bestimmter Zielsetzungen allein so schwierig wäre, sondern weil es ein höchst kompliziertes Unterfangen ist, die Meinungen unterschiedlicher Personen auf einen gemeinsamen Nenner zu bringen. Zwischenmenschliche Beziehungen sind unser täglich Brot: Der Chef muss seine Mannschaft formen, koordinieren und motivieren, so gut es geht, muss in den Köpfen seiner Mitarbeiter lesen und Nuancen erkennen können, gute Menschenkenntnis besitzen und akzeptieren, dass jeder auch eine private Seite hat. Jeder muss anders angefasst werden. Das ist eine enorme Leistung, die in keiner Tätigkeitsbeschreibung angemessene Erwähnung findet. Mit zunehmender Erfahrung verfeinert man natürlich die eigenen Führungsqualitäten; eine gewisse natürliche Bereitschaft und Neugier, sich mit anderen Mentalitäten auseinanderzusetzen, ist jedoch ebenfalls erforderlich.

Oftmals, wenn man die Dinge für zu selbstverständlich nimmt, entgleitet einem die Situation. Dann kommt es zu Streitigkeiten, Spannungen und Missverständnissen, die sich in Form von Krankheitsausfällen bemerkbar machen. Das ist eine Alarmglocke, die auf tiefgreifende Missstände hinweist. Ohne von sich zu verlangen, die Launen der eigenen Mitarbeiter stets im Griff haben zu müssen, reicht manchmal schon ganz wenig, um die Moral der Truppe wieder auf Vordermann zu bringen. Neben den regelmässigen internen Sitzungen spielen dabei informelle Momente eine wichtige Rolle. Eine gemeinsame Tasse Kaffee, ein kleiner Plausch mit der Sekretärin, ein Abendessen für die Mitarbeiter, ein freundliches Dankeschön für einen erwiesenen Gefallen, Lob für einen Mitarbeiter, der gute Arbeit geleistet hat, Verteidigung der Mitarbeiter gegen Kritik von aussen, auch wenn diese berechtigt war [8]. Man muss sich klar machen, dass die Mitarbeiter zunächst einmal Menschen sind, die nicht nur aus ökonomischer Notwendigkeit arbeiten, sondern in ihrer Arbeit auch ein identitäts- und sinnstiftendes Element sehen. Daher braucht jeder Mitarbeiter, um sich in seiner Aufgabe voll entfalten zu können, ein ehrliches Feedback vom Chef, und zwar sowohl in Form von Lob und Anerkennung als auch in Form von Tadel, sofern dieser begründet ist und in konkreter Weise vorgebracht wird.

Wie oft hätte ich etwas besser machen wollen, wie oft musste ich mit der Frustration leben, meinen Mitarbeitern nicht mehr geben zu können, und daher fürchten, kein guter Chef zu sein. Im Laufe der Jahre habe ich verstanden, dass man die Früchte seines Mühens erst in besonders schwierigen Situationen erntet, wenn nämlich die gesamte Mitarbeiterschaft geschlossen hinter ihrem Chef steht. Diese Beziehung beruht auf Gegenseitigkeit: Der Chef vermittelt Vertrauen, Erfahrung, Ruhe und Kompetenz, und die Mitarbeiter danken es ihm mit guter Arbeit und Loyalität. In einem Führungskurs für leitende Staatsangestellte hatten wir gelernt, Ziel des Chefs sei es, das Team so weit zu bringen, dass es den Chef nicht mehr braucht - weil es auch ohne ihn hervorragend funktioniert. Aus meiner Erfahrung kann ich sagen, dass dies ohne weiteres möglich ist, allerdings nur über begrenzte Zeiträume. Dann sind wieder Führung und Innovation vonnöten, die beiden Hauptqualitäten jedes Chefs.

\section{Aus dem Nähkästchen ...}

Heiteres und Kurioses bereichert den Alltag des Kantonsarztes. Ich bedauere es nur, über all die kleinen, seltsamen Begebenheiten, die - weitergeleitet von diversen anderen Ämtern - auf meinem Schreibtisch landeten, nicht Tagebuch geführt zu haben.

Ich erinnere mich gut an das Telefonat einer Dame aus Valcolla, die ein kantonsärztliches Eingreifen verlangte, weil der Esel ihres Nachbarn die ganze Nacht schrie und der Verlust ihrer Nachtruhe ihrer Gesundheit schade. Ich wandte mich daraufhin an die dortige Gemeinde und habe seitdem nichts mehr in dieser Angelegenheit gehört. Oder die Schulen, die verlangten, 
ich solle die Schliessung anordnen, weil es zu heiss sei oder zu feucht oder die Neonbeleuchtung nicht angemessen. Häufig verbargen sich hinter den vordergründigen Problemen ganz andere, hochverwickelte Angelegenheiten, die weit in die Vergangenheit zurückreichten und von denen ich nichts wusste.

Nicht selten war ich mit menschlichen Dramen konfrontiert: sozial abgeschottet lebende Menschen in von Unrat starrenden Wohnungen, bei denen einem schon das Betrachten der Fotos einen Schauer über den Rücken jagte. Diese Personen weigerten sich kategorisch, sich in eine entsprechende Einrichtung verbringen zu lassen. Das Ganze spielte sich in einer emotional stark aufgeladenen Atmosphäre ab, mit Familienstreitigkeiten und dem üblichen Interventionskarussell zwischen Gemeinde, Angehörigen, Hausarzt, Sozialarbeiter, psychosozialem Dienst usw. und keiner war für eine Entscheidung zuständig! Meine Position als Aussenstehender gestattete mir hier einen emotional unbeteiligten, analytischen Blick auf die Situation. Auf diese Weise brachten wir wieder etwas Ordnung in die Gedanken und legten gemeinsam fest, was nun der Reihe nach zu tun war.

\section{Der Esel des Nachbarn schrie die} ganze Nacht und raubte die

\section{Nachtruhe - der Kantonsarzt sollte einschreiten}

Die meisten dramatischen Momente erlebte ich jedoch in meiner Aufsichtsfunktion gegenüber den Mitarbeitern des Gesundheitswesens - hauptsächlich Ärzten. Diese Fälle endeten stets mit dem Suspendierungsverfahren oder dem Entzug der Zulassung zur Berufsausübung. Viele waren mit einem enormen Aufwand verbunden, von der Untersuchung der Strafakte (häufig ging das Verwaltungsverfahren, für das wir zuständig waren, mit einem Strafverfahren einher) über die Untersuchung der klinischen Dokumentation, die Gespräche mit Staatsanwälten, Anwälten und anderen Beteiligten, bis hin zum Umgang mit den Medien, für die solche Fälle immer ein gefundenes Fressen sind. Den intensivsten Einsatz erforderte jedoch stets die direkte Auseinandersetzung mit den Betroffenen, die sehr gefühlsgeladen und teilweise auch sehr schmerzhaft war. Geschichten, die jedem anderen genauso passieren könnten, mich selbst nicht ausgenommen. Schicksale, mit denen man konfrontiert wird.

Da gab es Ärzte bzw. Ärztinnen, die sich in eine leidenschaftliche Liebesaffäre mit ihren (mehr oder weniger willigen) Patientinnen oder Patienten stürzten und ihre Schäferstündchen als Kassenleistung abrechneten. Ärzte mit wahnhaften Grössenideen, die mittels
Fälschung und Betrug unglaubliche Vermögen und persönliche Macht anhäuften, um schliesslich unter den Trümmern ihrer eigenen Imperien zusammenzubrechen. Ärzte mit psychotischen Wahnvorstellungen, die - in der Überzeugung, die Welt vom Bösen zu erretten - ihren Patienten per Brief den Tod ankündigten und schliesslich in der Psychiatrie endeten. Andere wiederum landeten im Gefängnis und verbrachten dort geraume Zeit, ehe sie schliesslich entlastet werden konnten. Ich habe Ärzte gekannt, die aufgrund widriger Lebensumstände dem Alkohol verfielen und nur mit Mühe den Weg aus der Sucht fanden und wieder arbeitsfähig wurden. Ärzte, die illegale Drogen konsumierten, es zugaben und ihre Tätigkeit aufgeben mussten. Ärzte, die auf «Befehl» des Patienten («Herr Doktor, schreiben sie doch bitte, dass ...») sofort ein Attest ausstellten und sich nicht bewusst waren, welchen Schaden sie damit der Glaubwürdigkeit des gesamten Berufsstandes und der gesellschaftlichen Solidarität zufügten. Ärzte mit «hellseherischen Fähigkeiten», die im April bereits Krankschreibungen für einen Zeitraum im Juli ausstellten. Andere wiederum waren in derartige finanzielle Schwierigkeiten geraten, dass sie sich nur unter heftiger Selbstbemitleidung von einem ihrer PS-starken Luxusboliden trennten. Wieder andere Ärzte wurden infolge von Streitigkeiten mit den Krankenkassen selbst krank, vielen setzten Arbeit und Privatleben zu, bis sie buchstäblich ausgebrannt waren. Ich habe Ärzte gekannt, die infolge eines privaten Ereignisses eingeschüchtert und verängstigt waren. Ich habe Ärzte weinen sehen. Habe Ärzte sterben sehen. Alle diese Fälle zusammen bilden eine Minderheit - eine kleine Minderheit der über eintausend in unserem Kanton tätigen Ärzte. Eben diejenigen, deren persönliche Dramen, deren Probleme auf meinem Schreibtisch landeten.

Daneben habe ich aber auch andere Ärzte kennengelernt, die die eben angeführten Fälle zahlenmässig bei weitem übersteigen. Ärzte, die in ihrer Arbeit aufgehen und mit ihrem Leben zufrieden sind; ernsthafte Profis, die täglich im Einsatz sind, um die Gebrechen der Menschen zu lindern und Krankheiten zu heilen, die stets ein offenes Ohr für ihre Patienten haben und Unterstützung geben können. Oftmals mit grosser Zurückhaltung und in aller Stille.

\section{Ein Dekalog des Kantonsarztes}

Zum Abschluss meiner persönlichen Betrachtungen zum Berufsbild, zur Definition und zur Arbeit des Kantonsarztes möchte ich hier anhand einiger Verhaltensregeln ein paar Denkanstösse geben. Es liegt mir jedoch fern, irgendwelche Ratschläge zu erteilen. Eine alte Redensart lautet «Jeder macht seine Fehler allein». Die Idee zu dieser Auflistung von Verhaltensregeln entstand nach einer Publikation des Waadtländer Kollegen Jean Martin, der aufgrund seiner 27-jährigen Erfahrung als Kantonsarzt (1976-2003) einige Ratschläge für junge Kollegen formuliert hat [9]. 


\section{Dekalog des Kantonsarztes}

1. Gott hat uns zwei Ohren und nur einen Mund gegeben, damit wir mindestens doppelt so viel zuhören wie reden. (Chinesisches Sprichwort)

Zuhören, zuhören und nochmals zuhören. Stelle direkte Fragen, auch unangenehmer Art, aber stets mit Anstand. Setze dich mit jeder Frage ohne Stereotype und Vorurteile auseinander. Gehe nicht auf Beleidigungen ein, verbirg deine Verletzlichkeit. Zeige Mitgefühl und Transparenz, versetze dich in dein Gegenüber hinein. Jede Gereiztheit ist eine Niederlage.

2. Audiatur et altera pars. (Seneca)

Überstürze nichts, ehe du nicht die Meinung aller Beteiligten gehört hast. Bleibe unparteiisch und pflege einen höflichen Stil. Tritt mit Bestimmtheit auf, ohne unverrückbar auf deiner Meinung zu bestehen. Lüge nicht und rate anderen nicht zur Lüge. Vermeide Weissagungen und gewagte Versprechen.

3. Geduld ist eine Tugend der Starken. (anonym)

Sei geduldig. Gehe mit Ruhe und Mässigung vor, auch im besten Bestreben. Achte besonders auf die letzten Minuten eines Gesprächs, wenn die Konzentration nachlässt. Kontrolliere und halte deine Frustrationsschwelle auf hohem Niveau.

4. Erfahrung ist der Name, den die Menschen ihren Irrtümern geben. (Oscar Wilde)

Gib zu, wenn du dich geirrt hast. Aus Fehlern lernt man, sie bringen Erfahrung. Nur wer nichts tut, kann auch nichts falsch machen. Verstecke dich nicht hinter unglaubwürdigen Rechtfertigungen: Never complain, never explain! Schiebe nicht deinen Mitarbeitern die Verantwortung für deine Fehler in die Schuhe - du verlierst ihre Achtung.

5. Ne pas s'engager dans une guerre que I'on est sûr de perdre. (anonym)

Zeige Mut, aber keine Verwegenheit: Mut bedeutet nicht Verantwortungslosigkeit. Kommt Zeit, kommt Rat - Lösungen finden sich von selbst, wenn ihre Zeit gekommen ist. Viele Konflikte sind lichtscheu: Bei Licht betrachtet verflüchtigen sie sich. Denk immer an das Gebet des Theologen Reinhold Niebuhr: «Gott, gib mir die Gelassenheit, Dinge hinzunehmen, die ich nicht ändern kann, den Mut, Dinge zu ändern, die ich ändern kann, und die Weisheit, das eine vom anderen zu unterscheiden.»

6. Freunde kommen und gehen, Feinde sammeln sich an. (anonym)

Unterschätze nie dein Gegenüber. Vertraue niemandem blind, am wenigsten denen, die sich demonstrativ freundschaftlich geben und deine Arbeit über die Massen loben. Man kann es niemals allen recht machen und Kritik ist unvermeidlich. Das ist wie mit den Nebenwirkungen eines Arzneimittels: Um die gewünschte Wirkung zu erzielen, muss man sie in Kauf nehmen.

7. Man muss nicht recht haben, sondern die Mehrheit. (Flavio Guidotti)

Verliere nie den akademischen Ansatz aus den Augen, aber nimm es als gegeben, dass hier andere Regeln herrschen. Wir leben in keiner perfekten Welt. In einer Demokratie ist das Volk die oberste Instanz, auch wenn es im Unrecht ist. Ein Staatsdiener hat dies zu respektieren. Schon Winston Churchill bemerkte: «Die Demokratie ist die schlechteste aller Regierungsformen - abgesehen von all den anderen Formen, die von Zeit zu Zeit ausprobiert worden sind.»

8 Consensus is the Absence of Leadership. (Margaret Thatcher)

Respektiere das Recht auf abweichende Meinung, aber setze dich für deine Ideen ein. Und triff schliesslich eine Entscheidung im Rahmen deiner Kompetenz. Die schlechtestmögliche Entscheidung ist gar keine Entscheidung!

9 Für Freunde legt man das Gesetz aus, bei den anderen übt man es aus.

(Andrea Camilleri)

Gesetz, Recht und Gerechtigkeit sind drei unterschiedliche Dinge. Darüber sollte man sich möglichst früh im Klaren sein. In Entscheidungsfindungen fliessen viele divergierende Interessen ein, und der Richter verfügt über einen enormen Entscheidungsspielraum. Wahre deine Unparteilichkeit, die Verhältnismässigkeit deines Urteils und dein Gerechtigkeitsgefühl. Wenn das Gesetz dem Kantonsarzt Entscheidungsgewalt einräumt, ist dies beabsichtigt: Das Parlament weiss, dass der Kantonsarzt weder Jurist noch gerichtliche Instanz, sondern Arzt ist. Halte dich daher auch an die berufsethischen Normen und triff folgerichtige und klare Entscheidungen. Suche Rat bei den Juristen, aber nimm dir die Freiheit, ihn nicht zu befolgen.

10 Die Freiheit des Einzelnen endet dort, wo die Freiheit des Anderen beginnt. (Allgemeine Menschenrechtserklärung) [10] Die Freiheit des Einzelnen ist ein zentraler Wert der westlichen Kultur. Dennoch besteht daneben auch ein öffentliches Interesse, eine handelnde Öffentlichkeit, als deren Sprecher und Verteidiger der Kantonsarzt fungiert. Die Kunst des Erfolges liegt darin, diese beiden einander entgegengesetzten Rechte zu einem harmonischen Ganzen zu vereinen. «Est modus in rebus», empfiehlt der Dichter Horaz: Man muss in allen Dingen das richtige Mass finden. 


\section{Schlussfolgerungen}

Der Beruf des Kantonsarztes hat sich im Laufe der Jahrzehnte stark verändert. In der ärztlichen Vorstellung wird er oft zu Unrecht als ein zur Routine erstarrter, langweiliger Bürojob angesehen. Man hält ihn nicht einmal für einen ärztlichen Beruf, da ja weisser Kittel und Stethoskop fehlen, sondern betrachtet den Kantonsarzt als eine Art «Schreibtischarzt» - eine Bezeichnung, die mit kaum verhohlener Herablassung ausgesprochen wird. Dabei sieht die Realität ganz anders aus.

\section{Kantonsarzt ist ein Beruf, der an Bedeutung zunimmt, weil der Einfluss des Staates als regulierende Instanz im Gesundheitssystem zunimmt}

Heute handelt es sich um eine spannende, sich stetig entwickelnde, dynamische Tätigkeit, die den Kantonsarzt und sein Team tagtäglich mit neuen Herausforderungen konfrontiert. Ein Beruf, der an Bedeutung zunimmt, weil der Einfluss des Staates als regulierende Instanz im Gesundheitssystem tendenziell immer weiter zunimmt. Der Kantonsarzt kann durch sein Wirken - direkt oder indirekt über die Regierung - bedeutende Veränderungen im Gesundheitswesen auf den Weg bringen, Lösungen für unannehmbare Situationen schaffen, die Bevölkerung vor aufkommenden Krankheiten schützen und eine Kultur der Volksgesundheit fördern. Er kann die Gesundheitspolitik merklich beeinflussen - insbesondere, wenn es ihm gelingt, im Zusammenwirken mit anderen Experten innovative Lösungen und für die Regierung strategisch interessante Ansätze zu finden. Sein Wirken an der Nahtstelle zwischen Medizin und Politik gestattet ihm Einblicke in Stimmungslagen, Wesen und Logiken dieser beiden Welten, die eigentlich so wenig miteinander gemein haben und doch so stark miteinander verwoben sind.

Wir sind momentan Zeugen einer zunehmenden Verstaatlichung des Gesundheitswesens. Dies geschieht sowohl auf Betreiben der Ärzte selbst [11], die mehr Sicherheit verlangen (und dafür offenbar bereit sind,

In der nächsten SÄZ-Ausgabe folgt Kapitel 5 des Buchs, das eine Einführung in den Bereich Public Health darstellt. In diesem Teil sind auch die Schnittstellen zwischen Kantonsarzt und anderen Berufen im Bereich Public Health beschrieben. einen Teil ihrer Freiheit aufzugeben), als auch im Interesse der Politik, die eine bessere Kontrolle über die Gesundheitskosten anstrebt. Das Gesundheitssystem als für jeden Bürger garantierter Service public benötigt in der öffentlichen Verwaltung in zunehmendem Masse das Fachwissen von Medizinern, die in der Lage sind, gesundheitspolitische Entwicklungen auf der Basis des neuen Konzepts der Public Health voranzutreiben. Für den Beruf des Kantonsarztes bricht damit eine neue Zeit an.

\section{Literatur}

1 Martin J. Quand il s'agit d'apprécier, d'arbitrer, de conseiller... Bull Méd Suisses. 1998;79(12):480-3.

2 Baierlé JL. Le médecin cantonal: un généraliste de la santé publique. Bull Méd Suisses. 1989;70:101-3.

3 Cevey M. Médecine sociale et préventive et santé publique. Médecine \& Hygiène. 1967;25:1293-7.

4 Mit der verwendeten männlichen Form sind beide Geschlechter angesprochen.

5 Die formale Zuständigkeit für den Entzug der «Zulassung zum Arztberuf» liegt im Kanton Tessin beim Vorsteher des Gesundheits- und Sozialdepartements.

6 Es handelt sich hierbei um das Gesundheitsamt innerhalb des Gesundheits- und Sozialdepartements.

7 Mit Palast (oder it. «Palazzo») ist hier das «Amtsgebäude» gemeint. In der öffentlichen Verwaltung wird ein eigener Jargon gepflegt, der, ähnlich wie beim Militär, Ausdruck einer besonderen Kultur ist.

8 Meiner eigenen Erfahrung nach lässt sich mit hoher Toleranz gegenüber Fehlern das Beste aus den Mitarbeitern herausholen. Sie sind dann nicht gehemmt aus Angst, «in die Zeitung zu kommen». Die Angst davor, als Angehöriger der öffentlichen Verwaltung in die Schusslinie der Medien zu geraten, hat gravierende Auswirkungen auf die Arbeitseffizienz.

9 Martin J. Quand il s'agit d'apprécier, d'arbitrer, de conseiller... Bull Méd Suisses. 1998;79(12):480-3

10 In Artikel 29 heisst es: «Jeder ist bei der Ausübung seiner Rechte und Freiheiten nur den Beschränkungen unterworfen, die das Gesetz ausschliesslich zu dem Zweck vorsieht, die Anerkennung und Achtung der Rechte und Freiheiten anderer zu sichern und den gerechten Anforderungen der Moral, der öffentlichen Ordnung und des allgemeinen Wohles in einer demokratischen Gesellschaft zu genügen.» (Allgemeine Erklärung der Menschenrechte-Generalversammlung der Vereinten Nationen, 10. Dezember 1948).

11 Die 2009 ins Leben gerufene Volksinitiative «Ja zur Hausarztmedizin» möchte die Verantwortung des Staates in Bezug auf die Sicherung, Regulierung und Verwaltung dieses Berufsstands auf kantonaler und auf Bundesebene in der Verfassung verankern. 